\title{
E2District: Behaviour Demand Response ${ }^{\dagger}$
}

\author{
Julia Blanke*, Christian Beder, Emily Twomey, Sezen Aladag Ozdemir and Martin Klepal \\ People Behaviour \& Technology Integration Group, Nimbus Research Centre, Cork Institute of Technology, \\ Cork T12P928, Ireland; Christian.Beder@cit.ie (C.B.); Emily.Twomey@cit.ie (E.T.); \\ Sezen.Aladag@cit.ie (S.A.O.); Martin.Klepal@cit.ie (M.K.) \\ * Correspondence: Julia.Blanke@cit.ie \\ + Presented at the Sustainable Places 2017 (SP2017) Conference, Middlesbrough, UK, 28-30 June 2017.
}

Published: 3 November 2017

Keywords: behaviour demand response; supply side constrains; behavioural model

\section{Introduction}

This work describes the Behaviour Demand Response approach proposed in the context of the EU Horizon 2020 E2District project, which aims at achieving energy efficiency in District Heating and Cooling (DHC) systems applied in building blocks. Behaviour Demand Response (BDR) can be understood as the attempt to adjust the demand for power in accordance with supply side constraints, instead of adjusting the supply side alone without considering the potential flexibility of the demand. In contrast to this, typical current energy systems assume a rather strict distinction between a consumer driven demand side behaviour on one end and a matching supply side, usually provided by the energy grid, on the other end, with the primary objective of always satisfying consumer demands regardless of the constraints on the supply side. Demand side behaviour has always been modelled by energy suppliers to adapt production accordingly. There have been always incentive schemes in place to influence demand side behaviour like different tariffs according to supply side constrains. However, nowadays modern communication technology enables a much more granular and individualised approach. By including the building occupants in the energy optimisation process and considering their ability to interact with the environment as flexibility asset we are aiming to go beyond what purely technical solutions can achieve. The approach proposed here aims at modelling demand behaviour not only as an external factor to the system, but by incorporating the individual building occupant and his/her characteristics as an integral part of the system itself.

We propose to use a rigorous behavioural model to translate dynamic supply side constrains into adequate prosumer interactions, taking all relevant behavioural aspects like motivation, intention, personal traits and environment/context parameters into account. This model-based optimisation approach enables to target the right person at the right time in the right place with the right message/suggestion, dynamically involving the building occupant in the process as active participant/prosumer. This shifts the paradigm in the sense that occupants are not just consumers of energy anymore, but through their behaviour and how systems works today they can become producers of energy, too, the so called prosumers. This can either be through the availability of local production systems, like CHPs, but it is not limited to a situation where building occupants have production assets at their disposal. From the perspective of the overall energy grid, also the flexibility of their consumption creates a role as active assets in the systems, as soon as it is possible to engage with them and influence their behaviour according to supply side constraints. 


\section{Objectives of E2District}

The objectives of the E2District project are bringing together key stakeholders within the DHC value chain working together with the aim of developing an innovative set of tools for the optimisation of energy efficiency in the DHC industry. Furthermore the development of a District Simulation Platform is part of the project. This can be used as an Asset Portfolio Decision Support Tool to optimise DHC asset configuration and utilisation leading to more than $5 \%$ energy reduction. Another goal is to create Adaptive Control and Optimisation Methods for flexible production, storage and demand assets to develop algorithms for system fault diagnostics to enable cost effective maintenance. That will lead to an energy reduction of $10-20 \%$. Another major objective is to apply People Behaviour Demand Response by introducing additional behaviour flexibility to the system, keeping the human user in the loop estimating to save more than $5 \%$ of energy through this focus.

\section{Value Chain Innovation}

The work analyses how the value chain is further developed by allowing the demand to go in both directions, i.e., not only let energy suppliers react to consumer demand but also let prosumers respond to supply side constrains. This perspective is building a two way communication line. Therefore the classical demand-response paradigm towards a more efficient solution that integrates the prosumer as an active stakeholder into the value chain is achieved (see Figure 1). To obtain the aforementioned an integrated behavioural model is introduced. Based on well-established psychological theories a holistic model of behaviour is derived through the fusion of individual behavioural approaches in order to overcome each ones limitations. Thereby we aim to improve the overall outcome when it comes to motivate, influence and guide human behaviour (see Figure 2). By applying a model-driven approach supply side constrains are translated into prosumer interactions. Furthermore the model-driven approach serves to calibrate behavioural parameters based on real time measurements, enabling the fusion of heterogeneous input sources into a mathematical hidden Markov model, enabling the estimation and prediction of key parameters relevant to utilising the behaviour of building occupants as reliable flexibility assets also in the long-run.

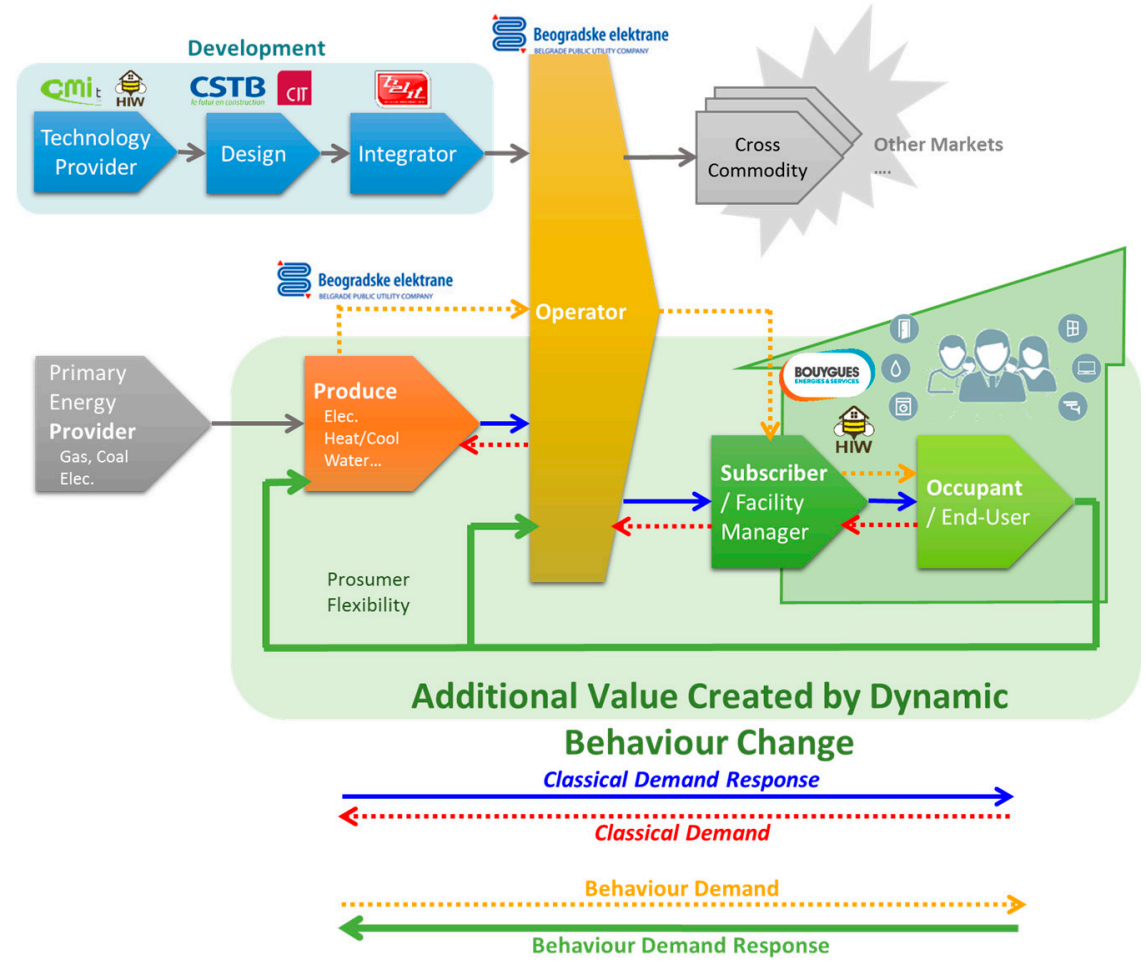

Figure 1. The Behaviour Demand Response value chain innovation. Value is not only created by reacting to consumer demand, but also by integrating the prosumer into the process. 


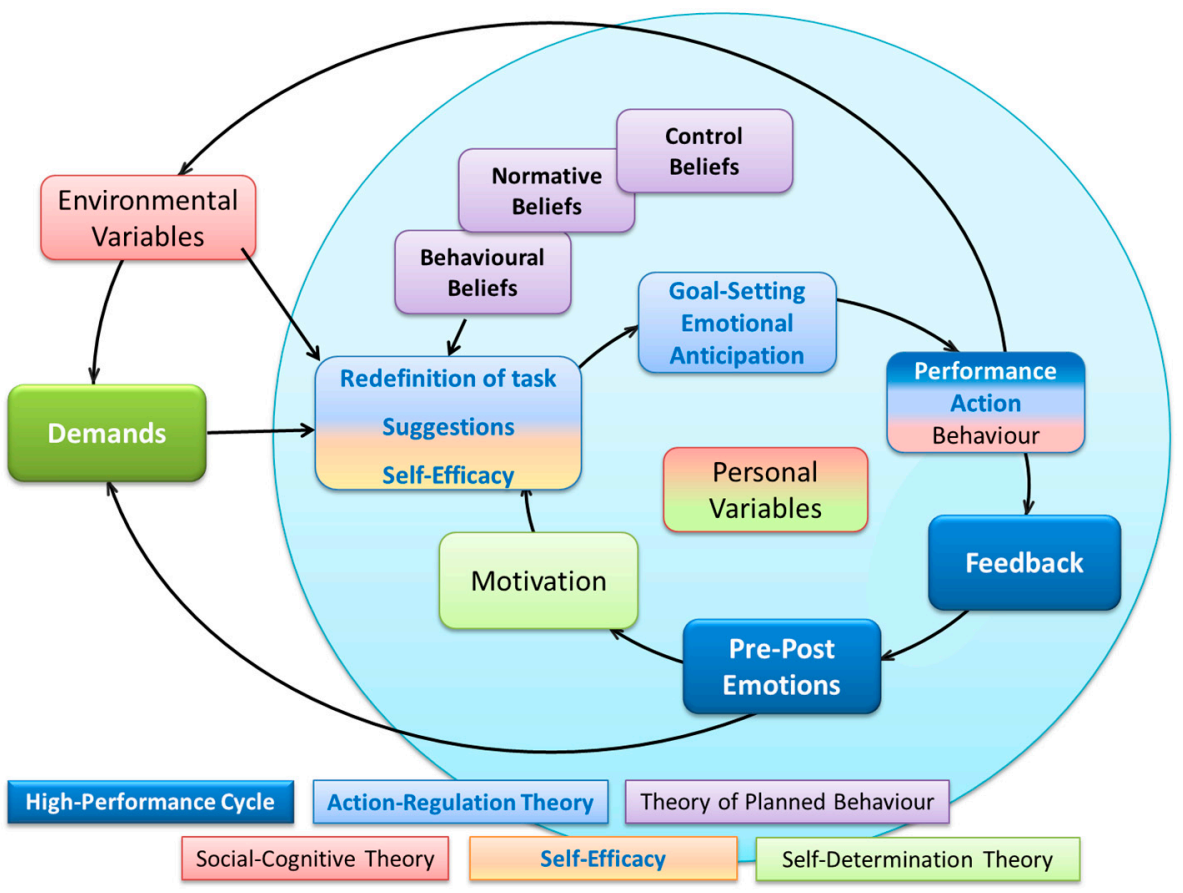

Figure 2. The proposed integrated behavioural model.

\section{E2District Behavioural Study-CIT Testbed}

The E2District project is executing a behavioural study with one of the testbeds at Cork Institute of Technology (CIT) in Ireland (see Figure 3). The first stage of the study involves the establishing of a research baseline by using technical sensors located throughout the campus testbed (i.e., 250 LoRa wireless sensors and actuators). Those sensors monitor room temperature, light intensity and air quality. Staff and student behaviour, in relation to temperature regulation, is also monitored i.e. opening and closing of doors and windows and adjustment of radiator valves. The second stage sees the introduction of the CIT Testbed mobile application, supported by interactive kiosks. The integrated behavioural model is used to configure the application to evaluate parameters that influence human behaviour such as environmental, personal, motivational and intentional factors. The aim of the application is to promote energy efficiency behaviour through contextualised personalised suggestions that enable users to influence their environment. The aforementioned is supposed to motivate occupants also in the long term. The application also includes useful features that benefit people in their daily tasks at CIT as user retention incentives.

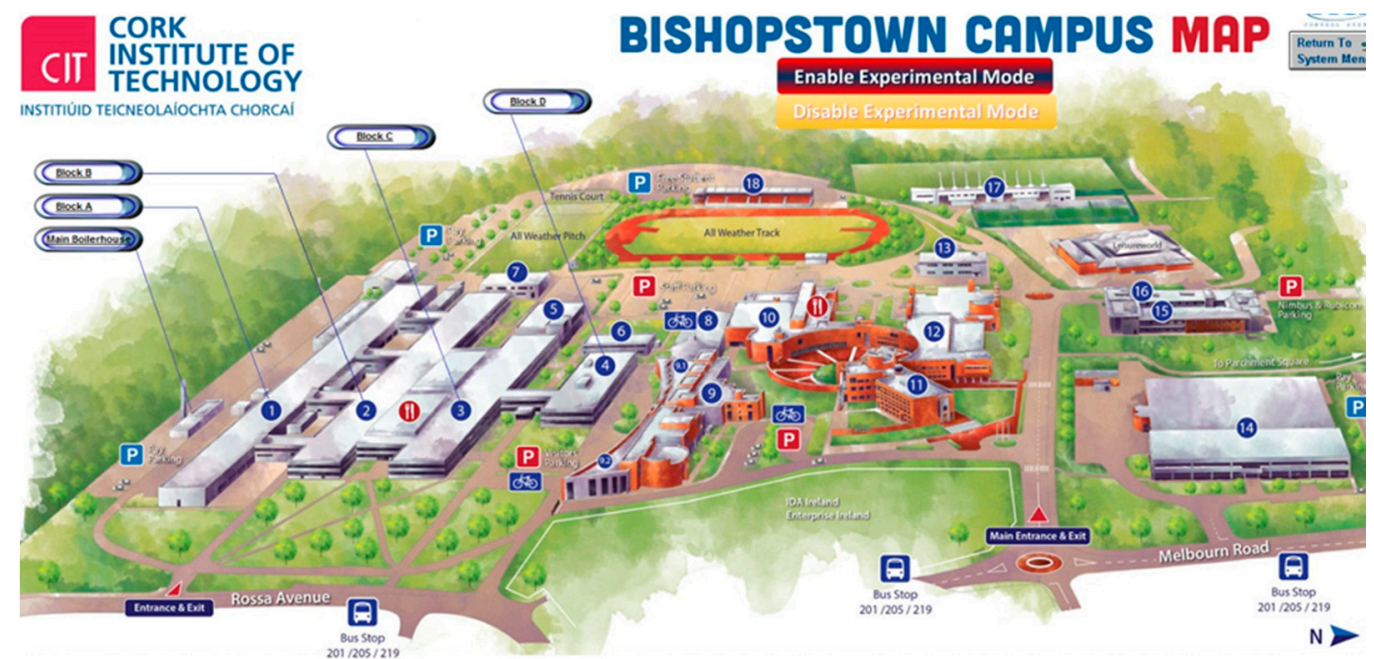

Figure 3. The Bishopstown Cork Institute of Technology Campus BDR Test Side. 


\section{Cyber Physical Systems Supports of BDR}

This cyber physical system is supporting BDR through a variety of approaches (see Figure 4). Important is to engage with the prosumer at all stages, while monitoring prosumers behaviour at all stages. We have LoRa sensors monitoring the influence of prosumers on their environment sending information to the supply side, which itself sends information to the prosumer. That is well embedded in the internet of things which are measuring environmental variables and their dynamic change. Furthermore the phone application interacts with the prosumer by making suggestions and giving feedback. All these interactions are based on a rigorous model of behaviour, enabling the estimation of behaviour relevant parameters, and providing the right message to the right person at the right time at the right place in order to optimally support the overall integrated energy optimisation.

\section{Cyber Physical System supports of BDR}

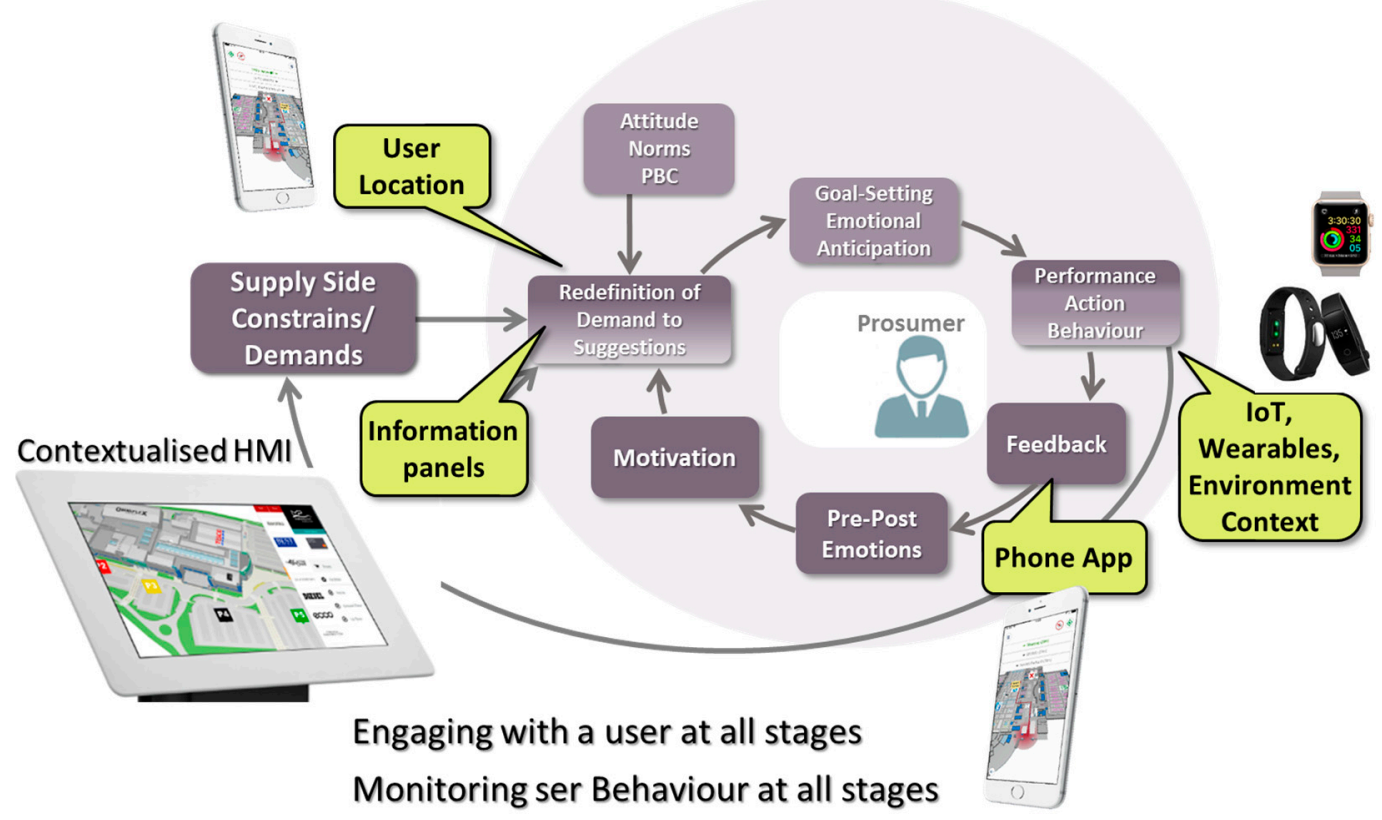

Figure 4. Cyber Physical System supports of Behaviour Demand Response.

\section{Conclusion}

The overall outcome is a behaviour demand response framework, enabling the integration of energy prosumers as active stakeholders into the energy supply value chain. They can be considered a flexibility asset to make the energy system more efficient. Therefore behavioural modelling enables optimisation of the overall process based on rigorous principles that allow the right person to be targeted with the right message at the right time in the right place, always adapting to changing dynamic context conditions. This approach is also applicable beyond the energy domain, in use cases such as building and smart city management, healthcare and personal health intervention and strategic tourism management.

Acknowledgement: This work has received funding from the European Union's Horizon 2020 Research and Innovation programme under Grant Agreement no. 696009.

C 2017 by the authors. Licensee MDPI, Basel, Switzerland. This article is an open access article distributed under the terms and conditions of the Creative Commons Attribution (CC BY) license (http://creativecommons.org/licenses/by/4.0/). 